\title{
The burden attributable to headache disorders in India: estimates from a community-based study in Karnataka State
}

\author{
Girish N. Rao', Girish B. Kulkarni², Gopalkrishna Gururaj', Lars J. Stovner ${ }^{3,4}$ and Timothy J. Steiner ${ }^{3,5^{*}}$
}

\begin{abstract}
Background: Headache disorders are common worldwide, causing pain and disability. India appears to have a very high prevalence of migraine, and of other headache disorders in line with global averages. Our objective was to estimate the burdens attributable to these disorders in order to inform health policy.

Methods: In a door-to-door survey, biologically unrelated adults (18-65 years) were randomly sampled from urban and rural areas of Bangalore and interviewed by trained researchers. The validated structured questionnaire enquired into several aspects of burden.

Results: Of 2,329 participants (non-participation rate 7.4\%), 1,488 (63.9\%; 621 male, 867 female) reported headache in the preceding year. Symptom burden was high. Migraine (1-year prevalence $25.2 \%$ ) occurred on average on 28 days/ year but, in $38.0 \%$ of cases (ie, $9.6 \%$ of adults), on $\geq 3$ days/month ( $\geq 10 \%$ of days). All causes of headache on $\geq 15$ days/ month (prevalence $3.0 \%$ ) occurred on a mean of 245 days/year. Both these and migraine were rated severe in intensity. Participants with headache lost $4.3 \%$ of productive time; those with migraine lost $5.8 \%$ (equating to $1.5 \%$ from the adult population). Lost paid worktime accounted for $40 \%$ of this, probably detracting directly from GDP. We estimated population-level disability attributable to migraine using the disability weight from GBD2010 for the ictal state (0.433). Mean disability per person with migraine was $1.8 \%$, reducing the functional capacity of the entire adult population by $0.46 \%$.

Fewer than one quarter of participants with headache had engaged with health-care services for headache in the last year. Actual expenditure on headache care was greatest among those with headache on $\geq 15$ days/month (especially probable medication-overuse headache), but otherwise not high. Expressed willingness to pay for effective treatment for headache was higher, signalling dissatisfaction with current treatments.
\end{abstract}

Conclusions: In Karnataka State, southern India, prevalent headache disorders, especially migraine, give rise to commensurately heavy burdens. Limited access to health care fails to alleviate these. Structured headache services, with their basis in primary care, are the most efficient, effective, affordable and equitable solution. They could be implemented within the health-care infrastructure of India and are likely to be cost-saving. This solution requires political will, itself dependent on awareness.

Keywords: Headache disorders, Migraine, Tension-type headache, Medication-overuse headache, Burden, Health policy, Willingness to pay, India, South-East Asia, Global campaign against headache

\footnotetext{
* Correspondence: t.steiner@imperial.ac.uk

${ }^{3}$ Department of Neuroscience, Norwegian University of Science and

Technology, Edvard Griegs Gate, NO-7491, Trondheim, Norway

${ }^{5}$ Division of Brain Sciences, Imperial College London, London, UK

Full list of author information is available at the end of the article
}

\section{它 Springer}

(c) 2015 Rao et al. Open Access This article is distributed under the terms of the Creative Commons Attribution 4.0 International License (http://creativecommons.org/licenses/by/4.0/), which permits unrestricted use, distribution, and reproduction in any medium, provided you give appropriate credit to the original author(s) and the source, provide a link to the Creative Commons license, and indicate if changes were made. 


\section{Background}

Primary headache disorders are not only among the commonest disorders in the world but also among the most burdensome $[1,2]$, causing pain, substantial disability, lost productive time and, consequentially, very large financial losses [3]. The Global Burden of Disease Study 2010 (GBD2010) reported tension-type headache (TTH) as the second most prevalent disorder worldwide and migraine as the third [1], but migraine far outweighs TTH as a cause of disability. GBD2010 ranked migraine as the seventh highest specific cause of disability in the world $[1,2]$, but this was an underestimate: data gathered since, and included in the subsequent Global Burden of Disease Study 2013 (GBD2013), elevated it to sixth [4]. Medication-overuse headache $(\mathrm{MOH})$, perhaps best regarded as a complication either of migraine or of TTH, since its occurrence is almost always as a consequence of mismanagement of one or the other, has also entered the top 20 causes of disability in GBD2013 [4].

In the context of India, these facts and statistics take on some significance. First, India has more than 1.2 billion people [5], over $16 \%$ of the world's population. Second, while headache disorders generally appear to be no less common in this country - at least in Karnataka State than elsewhere in the world [6], the prevalence of migraine is notably higher than the average reported from other countries [1, 7]. In our first report of this study, the 1-year prevalence of migraine was $25.2 \%$ and of TTH $35.1 \%$; the prevalence of all headache on $\geq 15$ days/month was $3.0 \%$ [6]. In GBD2010, the estimated worldwide prevalence of migraine was $14.7 \%$ and of TTH $20.8 \%$ [1]. The review of surveys by Stovner et al. estimated the mean global prevalence of all causes of headache on $\geq 15$ days/month at $3.0 \%$ [7]. It is likely, therefore, that very high burdens are attributable to headache in India; but, if so, they are largely disregarded by health services because knowledge of them is not available to inform health policy.

Across the world, knowledge for public-health policy is being gathered by a series of population-based studies supported by Lifting The Burden (LTB) [8, 9], a UKregistered non-governmental organisation conducting the Global Campaign against Headache [10] in official relations with the World Health Organization [11]. Methodology has been developed for this purpose [12, 13], focusing on the headache disorders of public-health importance: migraine, TTH, $\mathrm{MOH}$ and other causes of headache occurring on $\geq 15$ days/month. One study performed as part of this series was conducted in Karnataka State in southern India. The prevalence data from this study (referred to above) have been published already [6]; our purpose here, again to inform health policy, is to add estimates of burden attributable to these headache disorders, based on enquiries conducted during the survey.

\section{Methods}

The methodology of the study has been published in detail previously [14] and is described only briefly here. The institutional ethics committee of the National Institute for Mental Health and Neuro-Science (NIMHANS) approved the study protocol. Informed consent was obtained from all participants.

It was a cross-sectional survey, conducted during May to November 2009. It sampled urban and rural areas in and around Bangalore: Kempegowdanagara, an urban administrative ward in the city of Bangalore, and Uyamballi and Doddaaladahalli, two large villages located 75-80 Km from Bangalore. Trained interviewers travelled to these communities, selected households through multistage cluster sampling, called at each chosen household, listed all adult members (aged 18-65 years), randomly selected one and interviewed that person using a structured questionnaire. This instrument was an adaptation of the HARDSHIP questionnaire [13], translated into the local language (Kannada) in accordance with LTB's translation protocol for hybrid documents [15] and validated [14]. The headache screening question ("Have you had headache during the last year?") and diagnostic questions based on ICHD-II [16] were followed, for those reporting headache, by multiple question sets enquiring into various aspects of burden: symptom burden, lost productive time, health-care utilisation, personal expenditure on health care and willingness to pay for effective care. Any participant reporting more than one headache type was asked to focus only on the one that was subjectively the most bothersome for purposes of diagnosis and burden attribution.

Diagnoses were not made by the interviewers but later, by algorithm applied to the responses to the diagnostic questions. Participants with headache on $\geq 15$ days/month were first identified; among these, any with medication overuse were diagnosed as probable $\mathrm{MOH}(\mathrm{pMOH})$, the remainder as "other headache on $\geq 15$ days/month". The algorithm then applied ICHD-II criteria to all other cases in hierarchical sequence: first for migraine, then for TTH, then for probable migraine and finally for probable TTH. Cases remaining were considered unclassifiable. In the later analysis, migraine and probable migraine were grouped as all-migraine, and TTH and probable TTH as all-TTH.

\section{Statistics and analyses}

Data were entered into a secure database and statistical analyses were performed using EPI INFO [17] and SPSS 15 [18].

We used proportions, $95 \%$ confidence intervals (CIs), means and standard deviations (SDs), medians and quartiles to summarise the distributions of variables, and chisquared, Student's $t$-test or ANOVA to test for significance 
of differences. We used Pearson's coefficient to test for correlation between continuous variables. We set the level of significance at $5 \%$.

Headache frequency was reported as headache days/ year. Usual intensity was reported by participants on a 3-point categorical score as "not bad", "quite bad" and "very bad", which we equated to "mild", "moderate" and "severe"; these were converted to a numerical rating scale 1-3 and treated as continuous data to derive means $( \pm \mathrm{SD})$ and medians. We assessed lost productive time as a consequence of headache using the HALT questionnaire [19], enquiring separately into lost paid worktime (days lost from paid employment: questions 1 and 2) and lost household worktime (days lost from home chores: questions 3 and 4). The enquiry period was the preceding 3 months. To estimate the proportion of all productive time lost to each headache type, we assumed there were 240 working days/year. We then calculated the total working days/year available to all participants with each headache type (denominator) as $\mathrm{n} * 240$, and used the total reported days lost by them as numerator.

We estimated population-level disability from migraine using the disability weight (DW) from GBD2010 for the ictal state of migraine (0.433) [20]. We calculated mean time spent in the ictal state as a product of mean attack frequency and mean attack duration, and derived from this the proportion of total time that was spent in the ictal state. We multiplied this proportion by the DW to calculate individual disability, and the product by the prevalence of migraine to arrive at population-level disability.

\section{Results}

There were 2,329 participants (1,141 [49.0 \%] male, 1,188 [51.0 \%] female; mean age $38.0[ \pm 12.7]$ years; 1,103 [47.4\%] from rural areas, 1,226 [52.6 \%] urban). The overall participation rate was $92.6 \%$ (eligible population $n=2,514)$. The distributions of gender, age and habitation in the participating sample, described in detail previously, were comparable to those of the population of Karnataka [14].

Headache in the preceding year was reported by 1,488 participants (63.9\%; 621 male, 867 female), along with attributable symptom burden in terms of frequency and intensity. As reported previously, the age-standardised 1year prevalence of migraine was $25.2 \%$ and of TTH was $35.1 \%$; the age-standardised prevalence of all causes of headache on $\geq 15$ days/month was $3.0 \%$ and of $\mathrm{pMOH}$ was $1.2 \%$ [6]. Frequency for each of the headache types is presented in Table 1 . The majority of participants with migraine had relatively infrequent attacks, but a sizeable minority (227/597; $38.0 \%)$ had $\geq 3$ attacks/month. Overall, migraine was more frequent than $\mathrm{TTH}$, with a median of 2 days/month affected. Migraine was in almost all cases a moderate-to-severe headache, TTH mostly mild-to-moderate; headache on $\geq 15$ days/month, including $\mathrm{pMOH}$, was rated severe by two thirds of those affected (Table 1).

Lost productive time is shown in Table 2. It should be noted that many median values were zero (ie, at least half of people in the category of interest lost no productive time). For the episodic headaches this was so in most categories: for TTH all, and for migraine nearly all. Losses that were spread inconspicuously between paid and household worktime became apparent in total lost productive time. The SDs confirm that distributions were skewed, the usual pattern incorporating a severely affected minority.

Among participants with migraine, rural dwellers and females lost more time on average than urban dwellers and males, in both cases mostly accounted for by lost household worktime. Similar differences occurred among those with TTH. Much greater losses per person were attributable to the various causes of headache on $\geq 15$ days/ month, including $\mathrm{pMOH}$, although again several medians were zero.

Regardless of headache type, lost productivity correlated with headache frequency, both expressed in days/ year, albeit only weakly (Pearson correlations: lost paid worktime 0.271 ; lost household worktime 0.360 ; total lost productivity 0.402 ; all $p<0.0005$ ).

The estimates of all productive time lost to each headache type are in Table 3. All headache cost $4.3 \%$ of all productive time, females losing more than males $(p=0.0155)$. Migraine caused almost threefold higher losses than TTH (again females more than males, although not significantly $[p=0.1021])$, but those due to headache on $\geq 15$ days/month, and especially $\mathrm{pMOH}$, were far higher still.

In expected relation to lost productive time, we made an estimate of the population-level disability from migraine. Participants with migraine reported attacks on 28 days/year on average (Table 1), with a mean duration of $13.1( \pm 16.9)$ hours per attack; average time spent in the ictal state as a proportion of all time available to that person was therefore $4.2 \%\left([13.1 / 24]^{*}[28 / 365]^{*} 100\right)$. Multiplying this by the DW for the ictal state of migraine (0.433) [20], we calculated that each person with migraine carried an average $1.8 \%$ disability burden. Since the prevalence of migraine in the sample was $25.6 \%(597 / 2,329)$, this disability spread among the whole adult population was $0.46 \%$. In other words, migraine reduced the functional capacity of the entire adult population by $0.46 \%$.

We asked about consultations with health-care professionals specifically for headache in the last year. About one quarter of participants responded positively, the proportion depending on headache type. Of those with 
Table 1 Symptom burden: headache frequency and intensity by headache type

\begin{tabular}{|c|c|c|c|c|}
\hline & Migraine $(n=597)$ & Tension-type headache $(n=811)$ & $\mathrm{pMOH}(n=28)$ & Other headache on $\geq 15 \mathrm{~d} / \mathrm{m}(n=40)$ \\
\hline \multicolumn{5}{|c|}{ Headache frequency (headache days/year) } \\
\hline mean $\pm S D$ & $28 \pm 27$ & $17 \pm 20$ & $226 \pm 59$ & $259 \pm 78$ \\
\hline median & 24 & 10 & 216 & 240 \\
\hline \multicolumn{5}{|c|}{ Headache intensity $n(\%)$} \\
\hline "not bad" (=1) & $20(3.4)$ & $235(29.0)$ & $2(7.1)$ & $4(10.0)$ \\
\hline "quite bad" (=2) & $346(58.0)$ & $505(62.3)$ & $7(25.0)$ & $9(22.5)$ \\
\hline "very bad" (=3) & $231(38.7)$ & $71(8.8)$ & $19(67.9)$ & $27(67.5)$ \\
\hline mean $\pm S D$ & $2.4 \pm 0.5$ & $1.8 \pm 0.6$ & $2.6 \pm 0.7$ & $2.6 \pm 0.6$ \\
\hline median & 2.0 & 2.0 & 3.0 & 3.0 \\
\hline
\end{tabular}

$n$ number of participants with the headache type, $p M O H$ probable medication-overuse headache, $d / m$ days $/$ month

Table 2 Lost productive time attributed to headache for each headache type, by gender and by urban and rural habitation

\begin{tabular}{|c|c|c|c|c|c|c|}
\hline & \multicolumn{6}{|c|}{ Lost productive time (days per person per 3 months) } \\
\hline & \multicolumn{2}{|c|}{ Total lost productive time } & \multicolumn{2}{|c|}{ Lost paid worktime } & \multicolumn{2}{|c|}{ Lost household worktime } \\
\hline & Mean \pm SD & Median & Mean \pm SD & Median & Mean \pm SD & Median \\
\hline \multicolumn{7}{|l|}{ Migraine } \\
\hline male $(n=212)$ & $2.6 \pm 5.6$ & 1 & $1.3 \pm 4.8$ & 0 & $1.3 \pm 2.9$ & 0 \\
\hline female $(n=385)$ & $4.0 \pm 6.1^{* *}$ & 2 & $1.5 \pm 3.6$ & 0 & $2.5 \pm 4.5^{* * *}$ & 0 \\
\hline rural $(n=328)$ & $4.4 \pm 6.6$ & 3 & $1.6 \pm 3.8$ & 0 & $2.8 \pm 4.9$ & 1 \\
\hline urban $(n=269)$ & $2.3 \pm 4.8^{* * *}$ & 0 & $1.1 \pm 4.4$ & 0 & $1.2 \pm 2.4^{* * *}$ & 0 \\
\hline Total $(n=597)$ & $3.5 \pm 6.0$ & 2 & $1.4 \pm 4.1$ & 0 & $2.1 \pm 4.0$ & 0 \\
\hline \multicolumn{7}{|c|}{ Tension-type headache } \\
\hline male $(n=388)$ & $1.0 \pm 3.1$ & 0 & $0.4 \pm 1.8$ & 0 & $0.6 \pm 1.9$ & 0 \\
\hline female $(n=423)$ & $1.4 \pm 4.3$ & 0 & $0.4 \pm 2.1$ & 0 & $1.0 \pm 2.6^{*}$ & 0 \\
\hline rural $(n=416)$ & $1.5 \pm 4.8$ & 0 & $0.6 \pm 2.5$ & 0 & $1.0 \pm 2.8$ & 0 \\
\hline urban $(n=395)$ & $0.8 \pm 2.1^{* *}$ & 0 & $0.2 \pm 1.0^{* *}$ & 0 & $0.6 \pm 1.6^{*}$ & 0 \\
\hline Total $(n=811)$ & $1.2 \pm 3.8$ & 0 & $0.4 \pm 1.9$ & 0 & $0.8 \pm 2.3$ & 0 \\
\hline \multicolumn{7}{|c|}{ Probable medication-overuse headache } \\
\hline male $(n=4)$ & $18.3 \pm 21.3$ & 9 & $14.5 \pm 24.0$ & 4 & $3.8 \pm 4.8$ & 2.5 \\
\hline female $(n=24)$ & $13.1 \pm 17.7$ & 7.5 & $2.4 \pm 8.1$ & 0 & $10.7 \pm 11.5$ & 7 \\
\hline rural $(n=17)$ & $14.1 \pm 22.0$ & 5 & $6.5 \pm 14.7$ & 0 & $7.6 \pm 11.4$ & 5 \\
\hline urban $(n=11)$ & $13.5 \pm 9.7$ & 10 & $0.5 \pm 1.5$ & 0 & $13.0 \pm 10.0$ & 10 \\
\hline Total $(n=28)$ & $13.9 \pm 17.9$ & 8 & $4.1 \pm 11.8$ & 0 & $9.7 \pm 11.0$ & 6 \\
\hline \multicolumn{7}{|c|}{ Other headache on $\geq 15$ days/month } \\
\hline male $(n=14)$ & $7.3 \pm 9.4$ & 2 & $6.4 \pm 9.5$ & 1 & $0.9 \pm 3.5$ & 0 \\
\hline female $(n=26)$ & $10.9 \pm 9.9$ & 10 & $4.8 \pm 8.4$ & 0 & $6.0 \pm 6.4^{* *}$ & 5 \\
\hline rural $(n=19)$ & $12.1 \pm 11.6$ & 12 & $6.9 \pm 10.0$ & 0 & $5.1 \pm 6.7$ & 3 \\
\hline urban $(n=21)$ & $7.4 \pm 7.3$ & 5 & $4.0 \pm 7.2$ & 0 & $3.5 \pm 5.4$ & 0 \\
\hline Total $(n=40)$ & $9.6 \pm 9.7$ & 8 & $5.4 \pm 8.7$ & 0 & $4.3 \pm 6.0$ & 0 \\
\hline
\end{tabular}


Table 3 Estimated proportion (\%) of all productive time lost to all headache and each headache type

\begin{tabular}{|c|c|c|c|c|c|c|c|c|c|c|}
\hline & \multicolumn{10}{|c|}{ Estimated proportion } \\
\hline & \multicolumn{2}{|c|}{ All headache } & \multicolumn{2}{|c|}{ Migraine } & \multicolumn{2}{|c|}{ Tension-type headache } & \multicolumn{2}{|c|}{$\mathrm{pMOH}$} & \multicolumn{2}{|c|}{ Other headache on $\geq 15 \mathrm{~d} / \mathrm{m}$} \\
\hline & $n$ & $\%$ & $n$ & $\%$ & $n$ & $\%$ & $n$ & $\%$ & $n$ & $\%$ \\
\hline Total & 1,488 & $4.3>$ & 597 & 5.8 & 811 & 2.0 & 28 & 23.2 & 40 & 16.0 \\
\hline male & 621 & 3.0 & 212 & 4.3 & 388 & 1.7 & 4 & 30.5 & 14 & 12.2 \\
\hline female & 867 & 5.2 & 385 & 6.7 & 423 & 2.3 & 24 & 21.8 & 26 & 18.2 \\
\hline
\end{tabular}

$n$ number of participants in the category, $\mathrm{pMOH}$ probable medication-overuse headache, $d / m$ days/month

migraine, $30.3 \%$ had consulted, $84.0 \%$ of these in primary care (Table 4). The proportion was lowest among participants with TTH and far greater among those with headache on $\geq 15$ days/month.

Table 5 shows participants' estimates of their actual expenditure during the last 3 months on health care for headache, including consultations (if any) and medication. The pattern was similar: by far the greatest expenditures were claimed by those with headache on $\geq 15$ days/month, and, among these, by participants with $\mathrm{pMOH}$. Females spent more than males for all specific headache types, and rural expenditure was greater than urban except for $\mathrm{pMOH}$, although, with very wide variations, none of these differences was significant. The table also shows estimated expenditure as a percentage of total quarterly household income, since income must usually constrain what is spent. To make this calculation we multiplied reported monthly income by three. This analysis greatly highlights the expenditure by those with $\mathrm{pMOH}$.

Finally, we asked participants how much they would be willing to pay for effective treatment for their headache (meaning that it would no longer bother them), if it were available. This enquiry was intended as an overall measure of burden [12, 13]. Table 6 indicates a gradation: $\mathrm{pMOH}>$ other headache on $\geq 15$ days/month $>$ migraine $>$ TTH. It is noticeable (see bottom row) that considerably more would be paid for effective treatments for migraine or TTH than, reportedly, actually had been paid for current treatments.

\section{Discussion}

Our earlier report of this study showed that headache disorders were common in Karnataka State [6]. We observed that these data represented, for the time being, the best information available for the entire country and its more than 1.2 billion people [5]. The age-standardised 1-year prevalence of migraine, at $25.2 \%$, was well above the global average of $14.7 \%$ [1]. The estimated prevalence of all headache occurring on $\geq 15$ days/month was $3.0 \%$, equal to the global mean $[7,21]$, while that of $\mathrm{pMOH}$ was $1.2 \%$, within the range of most national estimates of $1-$ $1.5 \%$ [22].

The importance of these findings may seem selfevident for this very large population, but becomes much highlighted when burden data are added to them. Migraine, so highly prevalent, and the other headache disorders no less common than elsewhere in the world, generate commensurately heavy burdens. Among these are symptom burden - giving rise to health-care demand, disability, personal financial burden, and huge consequential losses in productive time - a large part of which translate into losses from gross domestic product (GDP). We discuss these, although not in this order.

Before doing so, we note the principal limitation inherent in this type of study. Our data were gathered in a cross-sectional survey, by questionnaire, from what we believe were a representative sample, but dependent on recall - in many cases over the preceding 3 months. The error associated with inexact recall has not been established [12], but there is no reason to suppose that it results in over- rather than underestimation.

\section{Symptom burden}

On all measures, migraine was a more burdensome headache than TTH, while the various causes of headache on $\geq 15$ days/month, especially $\mathrm{pMOH}$, were associated with greatest individual burden. In terms of symptoms, obviously the last occurred most frequently (on 245 days/year on average), but they were also, along with migraine, rated severe in intensity. They represent a

Table 4 Consultation with a health-care professional in the last year by headache type

\begin{tabular}{lcclc}
\hline & Migraine $(n=597)$ & Tension-type headache $(n=811)$ & $\mathrm{pMOH}(n=28)$ & Other headache on $\geq 15 \mathrm{~d} / \mathrm{m}(n=40)$ \\
\hline Any consultation made (\% of $\mathrm{n})$ & 30.3 & 16.6 & 78.6 & 72.5 \\
Primary care doctor (\%) & 84.0 & 81.6 & 72.7 & 51.7 \\
Specialist doctor (\%) & 13.2 & 13.6 & 27.3 & 41.4 \\
Others (\%) & 2.8 & 4.8 & 0 & 6.8 \\
\hline
\end{tabular}

$n$ number of participants with the headache type, $p M O H$ probable medication-overuse headache, $d / m$ days/month 
Table 5 Participants' estimates of their actual expenditure during the last 3 months on health care for headache by headache type

\begin{tabular}{lllll}
\hline Estimated expenditure (INR) & Migraine $(n=597)$ & Tension-type headache $(n=811)$ & $\mathrm{pMOH}(n=28)$ & Other headache on $\geq 15 \mathrm{~d} / \mathrm{m}(n=40)$ \\
\hline$\leq 100(\%)$ & 79.7 & 91.2 & 21.4 & 52.5 \\
$101-500(\%)$ & 15.2 & 6.9 & 53.6 & 35.0 \\
$501-1,000(\%)$ & 2.7 & 1.2 & 14.3 & 2.5 \\
$>1,000(\%)$ & 2.3 & 0.6 & 10.7 & 10.0 \\
Mean \pm SD & $112 \pm 307$ & $49 \pm 218$ & $532 \pm 710$ & $474 \pm 1,083$ \\
$\quad$ male & $71 \pm 143$ & $44 \pm 232$ & $289 \pm 383$ & $517 \pm 1,558$ \\
female & $134 \pm 366$ & $54 \pm 204$ & $573 \pm 748$ & $451 \pm 751$ \\
$\quad$ urban & $103 \pm 300$ & $34 \pm 177$ & $775 \pm 922$ & $330 \pm 657$ \\
$\quad$ rural & $119 \pm 314$ & $63 \pm 250$ & $375 \pm 501$ & $634 \pm 1,417$ \\
Median & 10 & 5 & 275 & 100 \\
\% of total household income & & & & $42.8 \pm 172.4$ \\
mean \pm SD & $6.7 \pm 49.9$ & $2.4 \pm 14.9$ & $32.9 \pm 76.9$ & 1.8 \\
median & 0.4 & 0.1 & 7.4 & 4.4 \\
\hline
\end{tabular}

INR Indian rupee, $n$ number of participants with the headache type, $p M O H$ probable medication-overuse headache, $d / m$ days $/$ month

very high symptom burden for $3 \%$ of the adult population. Migraine occurred on average more than twice a month, which itself is not an unusual finding in the world [7], but $38.0 \%$ of those with this disorder in Karnataka that is $9.6 \%$ of the adult population - were affected on $\geq 3$ days/month (at least $10 \%$ of days). This has important implications for health care, which we discuss later. Even if no account is taken of $\mathrm{TTH}$, the symptom burden of headache here is heavy.

\section{Lost productive time}

On most measures, females as a group reported greater burden than males, which was largely attributable to higher prevalence [6]. They also tended individually to report greater burden. However, this differential was not clearly seen in lost paid worktime, which almost certainly reflected the local culture of relatively few women being in paid employment. In estimating lost proportions of all productive time, we assumed there were 240 working days per year, which might not have been a fair assumption for household work, but there was no clear basis for any other assumption.

Lost productive time, perhaps especially including lost paid worktime, is one of the major burdens of headache. It has substantial implications not only for those directly affected but also for their dependents and society $[12,13]$. In this survey, while the heavy individual burdens attributable to $\mathrm{pMOH}$ and other headache on $\geq 15$ days/month are obviously of considerable importance to those affected, societal interest may focus on the $4.3 \%$ of all productive time lost to headache disorders collectively, given that this was the average for the nearly two thirds (63.9\%) of the population who reported headache. This meant the population as a whole aged 18-65 years (effectively the working population) lost $2.7 \%(4.3 * 0.639)$ of its entire productive time to headache - more than six days a year. This is an enormous loss. Those with migraine $(25.2 \%$ of the population) lost $5.8 \%$ of their productive time; they were therefore responsible for a $1.5 \%$ loss from the productive time of the population as a whole - nearly 4 days a year. Lost

Table 6 Participants' estimates of their willingness to pay (WTP) for effective treatment for headache, as an overall measure of burden, by headache type

\begin{tabular}{|c|c|c|c|c|}
\hline Reported amount willing to pay (INR) & $\begin{array}{l}\text { Migraine } \\
(n=597)\end{array}$ & Tension-type headache $(n=811)$ & $\mathrm{pMOH}(n=28)$ & Other headache on $\geq 15 \mathrm{~d} / \mathrm{m}(n=40)$ \\
\hline$\leq 100(\%)$ & 64.8 & 77.3 & 28.6 & 40.0 \\
\hline $101-500(\%)$ & 30.7 & 19.5 & 57.1 & 50.0 \\
\hline $501-1,000(\%)$ & 3.0 & 2.0 & 3.6 & 7.5 \\
\hline$>1,000(\%)$ & 1.5 & 1.2 & 10.7 & 2.5 \\
\hline Mean \pm SD & $206 \pm 452$ & $191 \pm 914$ & $435 \pm 577$ & $309 \pm 376$ \\
\hline Median & 100 & 50 & 200 & 200 \\
\hline Mean ratio of WTP to actual expenditure & $5.8 \pm 18.1$ & $8.8 \pm 22.3$ & $1.8 \pm 3.0$ & $2.4 \pm 3.7$ \\
\hline
\end{tabular}

INR Indian rupee, $n$ number of participants with the headache type, $p M O H$ probable medication-overuse headache, $d / m$ days $/$ month 
paid worktime accounted for $40 \%$ of this (Table 2), which might directly detract from GDP.

\section{Disability}

It is of interest to compare this reported lost productivity from migraine $(1.5 \%)$ with the disability estimate for the population attributable to those with migraine. This was considerably lower $(0.46 \%)$. It is important to recognise that lost productive time as captured by the HALT questionnaire [19] (and MIDAS, from which it derives [23]) is not a measure of disability but of behavioural response to impairment, correlating imprecisely with disability measures while being an important measure in its own right [13]. On the other hand, $5.8 \%$ (the proportion of lost productive time) is only modestly higher than $4.2 \%$ (the average proportion of all available time spent by those with migraine in the ictal state). It is in the nature of migraine that motivation and energy are lost, and that these symptoms, also expected to contribute to lost productivity, may for some time outlast what is described as the ictal state. Important questions therefore arise about the relationship between disability and productivity. In Zambia, with a somewhat lower migraine prevalence of $22.9 \%$ but higher attack frequency (41 days/year) and considerably longer reported mean duration $(36 \mathrm{~h})$, the same comparison found the disability estimate $(0.98 \%)$ to be similarly smaller than the reported total lost productivity (recalculated using 240 days as the denominator as $3.2 \%$ ) [24].

\section{Health care contact and expenditure}

We noted above that $38.0 \%$ of those with migraine $(9.6 \%$ of the adult population) were affected on $\geq 3$ days/month. This is often seen as a threshold for prophylactic medication, and therefore for requiring professional health care. In fact, among participants with migraine, not very far short of that proportion (30.3\%) had had contact with a health-care professional (mostly in primary care) at least once in the preceding year. Overall, slightly less than one quarter of participants with headache had seen a healthcare professional in the preceding year. Unsurprisingly, much higher proportions (about three quarters) of those with headache on $\geq 15$ days/month had done so, and were much more likely to have progressed to specialist care yet still they had headache on $\geq 15$ days/month, including $\mathrm{pMOH}$. We were not able to assess how effective the encounters with health-care professionals had been, or the benefits of any health care or advice offered, but it should be recognised that the measured burdens existed despite whatever health care was being provided.

Estimates (by participants) of actual expenditure on health care show that more was spent on migraine than on TTH, but the medians indicate that the majority of those affected, in either case, spent very little indeed.
The much higher expenditure by those with $\mathrm{pMOH}$ presumably reflected costs of medications, although they also consulted more.

\section{Willingness to pay}

WTP is considered to be an overall measure of burden $[12,13]$, although it is difficult to ground it. Furthermore, in similar studies in Zambia [24] and Georgia [25], WTP did not correlate with headache type or frequency, or with lost productive time. Clearly its determinants are complex. We are particularly cautious in drawing conclusions related to income, which in the social setting of India may be under-reported, especially by those close to the poverty line, in expectation of higher social security benefits.

What is possible is to make comparisons with participants' reported actual expenditure. While the latter might be inaccurate, participants would have had those reports in mind when considering WTP. It is therefore informative that the mean ratio of WTP to reported actual expenditure for migraine was close to six and for TTH close to nine. The difference between these probably reflected the much lesser amount expended on $\mathrm{TTH}$, while the low ratios for $\mathrm{MOH}$ (1.8) and other headache on $\geq 15$ days/month (2.4) were, even more probably, similarly influenced by the potentially impoverishing expenditures on these disorders. The clear implication of these comparisons is that a level of dissatisfaction existed with current treatments.

\section{Evidence of unmet need}

From all of these findings, the picture emerging in Karnataka is of a high proportion of people in a very large population with heavy symptom burdens attributable to headache, leading to disability and very substantial lost productivity. For whatever reasons, contact with healthcare professionals is achieved only by a minority. Apparent dissatisfaction with treatments probably reflects this.

This is a picture of unmet health-care need, because headache disorders are largely treatable [26]. Furthermore, because the lost-productivity (indirect) costs of untreated headache are enormous [3] and treatments can be highly cost-effective [27], there is an expectation that expenditure on treatment would be cost-saving overall [28].

\section{Conclusions}

In Karnataka State, in southern India, a very high prevalence of migraine and levels of TTH, pMOH and other headache on $\geq 15$ days/month similar to global averages give rise to commensurately heavy burdens. Among these are huge losses in productive time, a large part of which translate into losses from GDP. Care must be exercised in extrapolating beyond Karnataka, and we 
have discussed this issue previously [6]. But for the moment, while this remains the only study of its type in India, it is a fact that the data from it are the best available for all India.

A crucial finding is that the limited access to health care, which the study has demonstrated, fails to alleviate these burdens. Effective treatments exist [26], and could be made available through structured headache services, with their basis in primary care, which are the most efficient, effective, affordable and equitable solution [28]. Their implementation would be with the expectation of substantial cost-saving $[27,28]$. The three-tier model proposed by LTB for Europe [29] would readily adapt to the health-care infrastructure of India. This solution requires political will, which itself is dependent on awareness.

\section{Competing interests}

TJS and LS are directors and trustees of Lifting The Burden.

\section{Authors' contributions}

TJS and LJS conceived the study and drafted the protocol and study questionnaire, which were subsequently refined in discussion with GG. GG oversaw the study in Karnataka and took responsibility for quality control. GBK and GNR took charge of conduct of the study in the field, with GBK acting as principal investigator. GNR took responsibility for the statistical analysis. TJS drafted the manuscript. All authors reviewed and approved the final version.

\section{Acknowledgements}

This work was financially supported by Lifting The Burden, a UK-registered non-governmental organization conducting the Global Campaign against Headache in official relations with the World Health Organization. Our thanks are due to Dr Tarun Dua, World Health Organization, and to Dr R Srinivasa, Professor of Neurology, MS Ramaiah Hospitals, for their guidance and help. For diligent and meticulous data collection, we also thank Dr Kavita Rajesh, Junior Scientific Officer, and all field staff: Sri Lokesh M (team coordinator), Sri Damodhar, Sri Chandrashekar AG, Sri Ravichandra R, Sri Chandramohan KP and Sri Sombamadaiah. We are grateful to Sri Remees Raj, Data Manager, Centre for Public Health, NIMHANS, for statistical assistance.

\section{Author details \\ 'Department of Epidemiology, National Institute of Mental Health and Neuro Sciences (NIMHANS), Bangalore, India. ${ }^{2}$ Department of Neurology, National Institute of Mental Health and Neuro Sciences (NIMHANS), Bangalore, India. ${ }^{3}$ Department of Neuroscience, Norwegian University of Science and Technology, Edvard Griegs Gate, NO-7491, Trondheim, Norway. ${ }^{4}$ Norwegian Advisory Unit on Headaches, Nevrosenteret Øst, St Olavs University Hospital, Trondheim, Norway. ${ }^{5}$ Division of Brain Sciences, Imperial College London, London, UK}

Received: 29 June 2015 Accepted: 20 October 2015

Published online: 09 November 2015

\section{References}

1. Vos T, Flaxman AD, Naghavi M, Lozano R, Michaud C, Ezzati M, Shibuya K, Salomon JA, Abdalla S, Aboyans V, Abraham J, Ackerman I, Aggarwal R, Ahn SY, Ali MK, Alvarado M, Anderson HR, Anderson LM, Andrews KG, Atkinson C, Baddour LM, Bahalim AN, Barker-Collo S, Barrero LH, Bartels DH, Basáñez MG, Baxter A, Bell ML, Benjamin EJ, Bennett D et al. (2012) Years lived with disability (YLDs) for 1160 sequelae of 289 diseases and injuries 1990-2010: a systematic analysis for the Global Burden of Disease Study 2010. Lancet 380:2163-2196

2. Steiner TJ, Stovner L, Birbeck GL (2013) Migraine: the seventh disabler. J Headache Pain 14:1
3. Linde M, Gustavsson A, Stovner LJ, Steiner TJ, Barré J, Katsarava Z, Lainez JM, Lampl C, Lantéri-Minet M, Rastenyte D, Ruiz Dela Torre E, Tassorelli C, Andrée C (2012) The cost of headache disorders in Europe: the Eurolight project. Eur J Neurol 19:703-711

4. Vos T, Barber RM, Bell B, Bertozzi-Villa A, Biryukov S, Bolliger I, Charlson F, Davis A, Degenhardt L, Dicker D, Duan L, Erskine H, Feigin VL, Ferrari AJ, Fitzmaurice C, Fleming T, Graetz N, Guinovart C, Haagsma J, Hansen GM, Hanson SW, Heuton KR, Higashi H, Kassebaum N, Kyu H, Laurie E, Liang X, Lofgren K, Lozano R, Maclntyre MF et al. (2015) Global, regional, and national incidence, prevalence, and years lived with disability for 301 acute and chronic diseases and injuries in 188 countries, 1990-2013: a systematic analysis for the Global Burden of Disease Study. Lancet. 2013;386:743-800.

5. Government of India (2011) 2011 census data. Available at http://censusindia.gov.in/ (last accessed 6 November 2015).

6. Kulkarni GB, Rao GN, Gururaj G, Stovner LJ, Steiner TJ (2015) Headache disorders and public ill-health in India: prevalence estimates in Karnataka State. J Headache Pain 16:67

7. Stovner LJ, Hagen K, Jensen R, Katsarava Z, Lipton RB, Scher Al, Steiner TJ, Zwart J-A (2007) The global burden of headache: a documentation of headache prevalence and disability worldwide. Cephalalgia 27:193-210

8. Steiner TJ (2004) Lifting the burden: The global campaign against headache. Lancet Neurol 3:204-205

9. Steiner TJ (2005) Lifting The Burden: the global campaign to reduce the burden of headache worldwide. J Headache Pain 6:373-377

10. Steiner TJ, Birbeck GL, Jensen R, Katsarava Z, Martelletti P, Stovner $L$ (2010) Lifting The Burden: the first 7 years. J Headache Pain 11:451-455

11. Steiner TJ, Birbeck GL, Jensen R, Katsarava Z, Martelletti P, Stovner LJ (2011) The Global Campaign, World Health Organization and Lifting The Burden: collaboration in action. J Headache Pain 12:273-274

12. Stovner $L$, Al Jumah $M$, Birbeck $G L$, Gururaj $G$, Jensen R, Katsarava Z, Queiroz LP, Scher Al, Tekle-Haimanot R, Wang SJ, Steiner TJ (2014) The methodology of population surveys of headache prevalence, burden and cost: Principles and recommendations from the Global Campaign against Headache. J Headache Pain 15:5

13. Steiner TJ, Gururaj G, Andrée C, Katsarava Z, Ayzenberg I, Yu S-Y, Al Jumah M, Tekle-Haimanot R, Birbeck GL, Herekar A, Linde M, Mbewe E, Manandhar K, Risal A, Jensen R, Queiroz LP, Scher Al, Wang SJ, Stovner L (2014) Diagnosis, prevalence estimation and burden measurement in population surveys of headache: presenting the HARDSHIP questionnaire. J Headache Pain 15:3

14. Rao GN, Kulkarni GB, Gururaj G, Rajesh K, Subbakrishna DK, Steiner TJ, Stovner LJ (2012) The burden of headache disorders in India: methodology and questionnaire validation for a community-based survey in Karnataka State. J Headache Pain 13:543-550

15. Peters M, Bertolote JM, Houchin C, Kandoura T, Steiner TJ (2007) Translation protocol for hybrid documents. J Headache Pain 8(suppl 1):S45-S47

16. Headache Classification Subcommittee of the International Headache Society (2004) The International Classification of Headache Disorders: 2nd edition. Cephalalgia 24 Suppl 1:9-160

17. Dean AG, Arner TG, Sunki GG, Friedman R, Lantinga M, Sangam S, Zubieta JC, Sullivan KM, Brendel KA, Gao Z, Fontaine N, Shu M, Fuller G, Smith DC, Nitschke DA, Fagan RF (2007) Epi Info ${ }^{\mathrm{TM}}$, a database and statistics program for public health professionals, version 3.5.1. Centers for Disease Control and Prevention, Atlanta GA, USA

18. SPSS Inc (2006) Statistical package for social sciences, USA version 15.0. SPSS Inc, Chicago IL, USA

19. Steiner TJ (2007) The HALT and HART indices. J Headache Pain 8(Suppl 1):S22-S25

20. Salomon JA, Vos T, Hogan DR, Gagnon M, Naghavi M, Mokdad A, Begum N, Shah R, Karyana M, Kosen S, Farje MR, Moncada G, Dutta A, Sazawal S, Dyer A, Seiler J, Aboyans V, Baker L, Baxter A, Benjamin EJ, Bhalla K, Bin Abdulhak A, Blyth F, Bourne R, Braithwaite T, Brooks P, Brugha TS, BryanHancock C, Buchbinder R, Burney P et al. (2012) Common values in assessing health outcomes from disease and injury: disability weights measurement study for the Global Burden of Disease Study 2010. Lancet 380:2129-2143

21. Jensen R, Stovner LJ (2008) Epidemiology and comorbidity of headache. Lancet Neurol 7:354-61

22. Westergaard ML, Hansen EH, Glumer C, Olesen J, Jensen RH (2014) Definitions of medication-overuse headache in population-based studies and their implications on prevalence estimates: A systematic review. Cephalalgia 34:409-25 
23. Stewart WF, Lipton RB, Kolodner KB, Sawyer J, Lee C, Liberman JN (2000) Validity of the Migraine Disability Assessment (MIDAS) score in comparison to a diarybased measure in a population sample of migraine sufferers. Pain 88:41-52

24. Mbewe E, Zairemthiama P, Paul R, Birbeck GL, Steiner TJ (2015) The burden of primary headache disorders in Zambia: national estimates from a population-based door-to-door survey. J Headache Pain 16:36

25. Lampl C, Steiner TJ, Mueller T, Mirvelashvili E, Djibuti M, Kukava M, Dzagnidze A, Jensen R, Stovner LJ, Katsarava Z (2012) Will (or can) people pay for headache care in a poor country? J Headache Pain 13:67-74

26. Steiner TJ, Paemeleire K, Jensen R, Valade D, Savi L, Lainez MJA, Diener H-C, Martelletti P, Couturier EGM (2007) European principles of management of common headache disorders in primary care. J Headache Pain 8(Suppl 1):S3-21

27. Linde M, Steiner TJ, Chisholm D (2015) Cost-effectiveness analysis of interventions for migraine in four low- and middle-income countries. J Headache Pain 16:15

28. World Health Organisation and Lifting The Burden (2011) ATLAS of headache disorders and resources in the world 2011. World Health Organization, Geneva

29. Steiner TJ, Antonaci F, Jensen R, Lainez JMA, Lanteri-Minet M, Valade D (2011) Recommendations for headache service organisation and delivery in Europe. J Headache Pain 12:419-26

\section{Submit your manuscript to a SpringerOpen ${ }^{\circ}$ journal and benefit from:}

- Convenient online submission

- Rigorous peer review

- Immediate publication on acceptance

- Open access: articles freely available online

- High visibility within the field

- Retaining the copyright to your article 